\title{
Coulomb scattering with remote continuum states in quantum dot devices
}

\author{
R. Wetzler, A. Wacker, and E. Schöll \\ Institut für Theoretische Physik, Technische Universität Berlin, Hardenbergstr. 36, 10623 Berlin, Germany
}

(Dated: November 20, 2018)

\begin{abstract}
Electron capture and emission by Coulomb scattering in self-assembled quantum dot (QD) devices is studied theoretically. While the dependence of the Coulomb scattering (Auger) rates on the local wetting layer electron density has been a topic of intense research, we put special interest on the remote scattering between QD electrons and continuum electrons originating from a quantum well, doped bulk layers or metal contacts. Numerical effort is made to include all microscopic transitions between the Fermi distributed continuum states. The remote Coulomb scattering is investigated as a function of the electron density, the distance from the QDs and the temperature. Our results are compared with experimental observations, considering lifetime limitations in QD memory structures as well as the electron emission in pn-diodes.
\end{abstract}

PACS numbers: 73.21.La,73.63.Kv,85.35.Be

\section{INTRODUCTION}

The controversial discussions about the nature of the electron dynamics in quantum dots 1] (QDs) show that this topic is still not fully understood. Different electronphonon coupling regimes leading to a multi-phonon process [2, 3, 4] or a polaron decay [5, 6] have been proposed. Especially for a multi-phonon process a so-called bottleneck effect 7] is expected due to the discrete QD density of states.

On the other hand it has been shown theoretically [8, 9, 10] as well as experimentally 11, 12] that electron capture and relaxation by Coulomb scattering (Auger effect) with electrons in the wetting layer can be very efficient for large enough densities. Furthermore, an Augertype process can overcome the bottleneck due to the continuous energy dispersion in the wetting layer.

The depletion of the wetting layer density by an electric field [13] has been shown to reduce the relaxation rates. Similarly, the electron density in the vicinity of the QDs is reduced in a pn diode by the depletion layer within a DLTS experiment [14, 15, 16]. Electrons which are candidates for a Coulomb scattering process are not located at the QDs anymore, but at some distance. Therefore we are interested in Coulomb scattering with remote continuum electrons of a quantum well and bulk contact regions as a function of the distance from the QDs.

We propose a theory to calculate the transition probabilities between two QD and two continuum states. Debye screening effects are included according to the threedimensional Poisson equation. From the two-particle scattering probability we calculate the total scattering probability of the electron relaxation within a QD (Auger process) and the excitation from a lower state into an excited state (impact ionization or impact excitation) by a numerical integration over the Fermi distributed continuum states.

\section{THEORY}

\section{A. General}

The band diagram of a QD and a remote quantum well and a bulk region defined by the doping profile is sketched in Fig. 11 We assume that the 2DEG and the 3DEG are confined by infinitely high barriers. The transition probability per unit time between the QD states $i$ and $j$, and the continuum states $\boldsymbol{k}$ and $\boldsymbol{k}^{\prime}$ is calculated by Fermi's Golden Rule

$$
W_{i \boldsymbol{k} \rightarrow j \boldsymbol{k}^{\prime}}=\frac{2 \pi}{\hbar}\left|M_{i \boldsymbol{k} \rightarrow j \boldsymbol{k}^{\prime}}\right|^{2} \delta\left(E_{\mathrm{ini}}-E_{\mathrm{fin}}\right),
$$

where $E_{\text {ini }}$ and $E_{\text {fin }}$ are the initial and final energies. The matrix element is given by

$M_{i \boldsymbol{k} \rightarrow j \boldsymbol{k}^{\prime}}=\iint \mathrm{d}^{3} \boldsymbol{r} \mathrm{d}^{3} \boldsymbol{r}^{\prime} \psi_{\boldsymbol{k}^{\prime}}^{*}(\boldsymbol{r}) \psi_{j}^{*}\left(\boldsymbol{r}^{\prime}\right) U\left(\boldsymbol{r}, \boldsymbol{r}^{\prime}\right) \psi_{i}\left(\boldsymbol{r}^{\prime}\right) \psi_{\boldsymbol{k}}(\boldsymbol{r})$,

where $\psi_{i}, \psi_{i}, \psi_{\boldsymbol{k}}$ and $\psi_{\boldsymbol{k}^{\prime}}$ symbolize the respective wave functions [17]. $U$ represents the effective Coulomb potential, which can be written in a two-dimensional Fourier representation as

$$
U\left(\boldsymbol{r}, \boldsymbol{r}^{\prime}\right)=\frac{1}{A} \sum_{\boldsymbol{q}^{\prime}} U\left(q^{\prime}, z, z^{\prime}\right) e^{i \boldsymbol{q}^{\prime}\left(\boldsymbol{r}_{\|}-\boldsymbol{r}_{\|}^{\prime}\right)},
$$

with

$$
U\left(q^{\prime}, z, z^{\prime}\right)=\frac{e^{2} e^{-q^{\prime}\left|z-z^{\prime}\right|}}{2 q^{\prime} \epsilon_{0} \epsilon_{r} \epsilon_{e}\left(q^{\prime}, z, z^{\prime}\right)} .
$$

Here, $\epsilon_{r}$ and $\epsilon_{0}$ are the relative and absolute permittivities, $e>0$ is the elementary charge, $A$ is the normalization area, $z, z^{\prime}$ are the coordinates in growth direction, $\boldsymbol{r}_{\|}, \boldsymbol{r}_{\|}^{\prime}$ are the in-plane coordinates, and $\boldsymbol{q}^{\prime}$ are two-dimensional wave vectors with $\left|\boldsymbol{q}^{\prime}\right|=q^{\prime}$. The dielectric function $\epsilon_{e}$ accounts for static screening effects of free electron densities. The evaluation of the matrix elements (2) depends on the QD wave functions, which themselves depend crucially on the QD shape. In order 


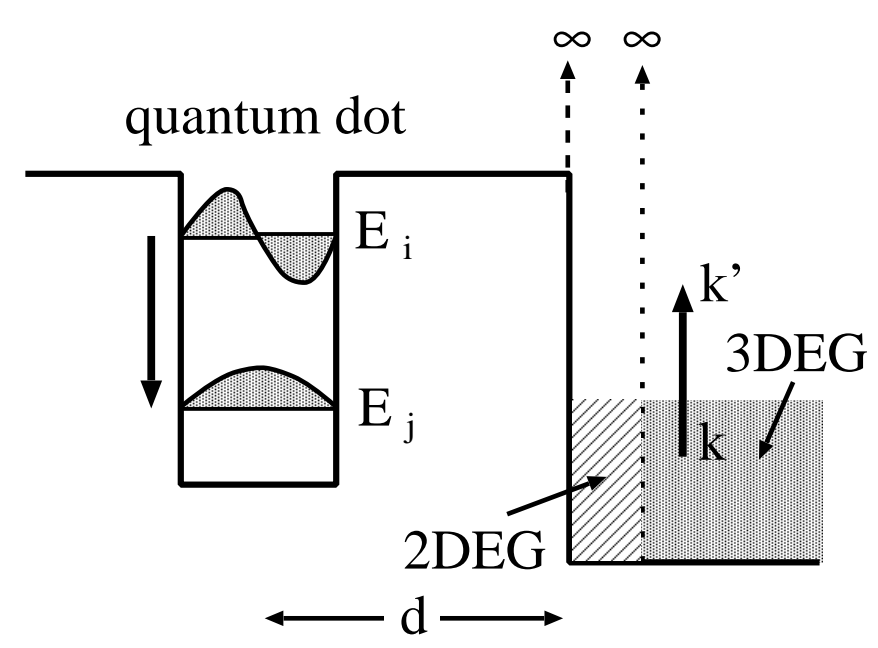

FIG. 1: Sketched band diagram showing the remote Coulomb scattering with two- and three-dimensional continuum electrons

to estimate the integral, we note that the wave functions $\psi_{i}(\boldsymbol{r})$ and $\psi_{j}(\boldsymbol{r})$ are orthogonal and thus the lowest order contribution results from the dipole moment $-e \mathbf{a}$ of the effective charge density $-e \psi_{i}^{*}(\boldsymbol{r}) \psi_{j}(\boldsymbol{r})$. We approximate this dipole moment by two localized charges of opposite sign and thus obtain

$$
\int \mathrm{d}^{3} \boldsymbol{r}^{\prime} \psi_{j}^{*}\left(\boldsymbol{r}^{\prime}\right) U\left(\boldsymbol{r}, \boldsymbol{r}^{\prime}\right) \psi_{i}\left(\boldsymbol{r}^{\prime}\right) \approx U\left(\boldsymbol{r}, \frac{\boldsymbol{a}}{2}\right)-U\left(\boldsymbol{r},-\frac{\boldsymbol{a}}{2}\right),
$$

where the center position of the QD is chosen as $\boldsymbol{r}^{\prime}=0$. The total transition probability per unit time from a QD state $i$ into a QD state $j$ is then obtained from an integration over the continuum states

$$
W_{i \rightarrow j}=\left(\frac{2 \Omega}{(2 \pi)^{n}}\right)^{2} \iint \mathrm{d}^{n} \boldsymbol{k} \mathrm{d}^{n} \boldsymbol{k}^{\prime} W_{i \boldsymbol{k} \rightarrow j \boldsymbol{k}^{\prime}} f_{k}\left[1-f_{k^{\prime}}\right],
$$

where $f_{k}$ and $f_{k^{\prime}}$ are the Fermi distribution functions of the initial and final continuum states. For a twodimensional electron gas (2DEG) we substitute $n=2$ and $\Omega=A$, and $n=3$ and $\Omega=A L$ for a threedimensional electron gas (3DEG), where $L$ is a (large) normalization length.

\section{B. Two-dimensional electron gas}

In order to find an expression for the dielectric function $\epsilon_{e}$ for a two-dimensional electron gas positioned at $z=d$, we consider an elementary charge positioned at $z=0$, corresponding to an external potential with the Fourier components

$$
U_{\text {ext }}(q, z)=\frac{e^{2} e^{-q|z|}}{2 q \epsilon_{0} \epsilon_{r}} .
$$

For the induced potential $U_{\text {ind }}$ originating from the induced electron density $\delta n_{2 \mathrm{D}}(q)$ in the quantum well layer the Fourier components of the three-dimensional Poisson equation [16] read as

$$
-q^{2} U_{\text {ind }}(q, z)+\partial_{z}^{2} U_{\text {ind }}(q, z)=-\frac{e^{2} \delta n_{2 \mathrm{D}}(q)}{\epsilon_{0} \epsilon_{r}} \delta(z-d),
$$

with the solution

$$
\delta U_{\text {ind }}(q, z)=\frac{e^{2}}{2 \epsilon_{0} \epsilon_{r} q} \delta n_{2 \mathrm{D}}(q) e^{-q|z-d|} .
$$

Within the Debye screening approach, $\delta n_{2 \mathrm{D}}(q)$ is given by

$$
\delta n_{2 \mathrm{D}}(q)=-\frac{\partial n_{2 \mathrm{D}}}{\partial E_{F}}\left[U_{\mathrm{ind}}(q, d)+U_{\mathrm{ext}}(q, d)\right]
$$

Assuming a single 2DEG quantization energy $E_{2 \mathrm{D}}, n_{2 \mathrm{D}}$ is given by

$$
n_{2 \mathrm{D}}=\frac{m^{*} k_{B} T}{\pi \hbar^{2}} \ln \left(1+\exp \left(\frac{E_{F}-E_{2 \mathrm{D}}}{k_{B} T}\right)\right),
$$

where $E_{F}$ is the Fermi energy, $m^{*}$ is the effective mass, $T$ is the temperature and $k_{B}$ is Boltzmann's constant, leading to the dielectric function for a $2 \mathrm{DEG}$

$$
\epsilon_{e}^{-1}(q, d)=\frac{U_{\text {ext }}(q, d)+U_{\text {ind }}(q, d)}{U_{\text {ext }}(q, d)}=\left[1+\frac{\lambda_{2 \mathrm{D}}}{q}\right]^{-1},
$$

with the inverse screening length

$$
\lambda_{2 \mathrm{D}}=\frac{e^{2} m^{*}}{2 \pi \epsilon_{0} \epsilon_{r} \hbar^{2}}\left[1+\exp \left(\frac{E_{2 \mathrm{D}}-E_{F}}{k_{B} T}\right)\right]^{-1}
$$

Inserting the two-dimensional Fourier expansion of the Coulomb potential into Eq. (5) and using plane waves for the 2DEG states, we find

$$
\begin{aligned}
& \left|M_{i \boldsymbol{k} \rightarrow j \boldsymbol{k}^{\prime}}^{2 \mathrm{DEG}}\right|^{2}=\mid \int_{A} \frac{e^{-i \boldsymbol{k}^{\prime} \boldsymbol{r}_{\|}}}{\sqrt{A}} \sum_{\boldsymbol{q}^{\prime}} \frac{e^{2} e^{-q^{\prime} d+i \boldsymbol{q}^{\prime} \boldsymbol{r}_{\|}}}{2 \epsilon_{0} \epsilon_{r} A\left(q_{\|}^{\prime}+\lambda_{2 \mathrm{D}}\right)} \\
& \times\left.\left[e^{q^{\prime} \frac{a_{z}}{2}+i \boldsymbol{q}^{\prime} \frac{\boldsymbol{a}_{\| 1}}{2}}-e^{-q^{\prime} \frac{a_{z}}{2}-i \boldsymbol{q}^{\prime} \frac{\boldsymbol{a}_{\|}}{2}}\right] \frac{e^{i \boldsymbol{k} \boldsymbol{r}_{\|}}}{\sqrt{A}} \mathrm{~d}^{2} r_{\|}\right|^{2} .
\end{aligned}
$$

$\boldsymbol{a}_{z}$ and $\boldsymbol{a}_{\|}$are the $z$ - and parallel components of $\boldsymbol{a}, \boldsymbol{k}$ and $\boldsymbol{k}^{\prime}$ are the two-dimensional wave vectors of the initial and final $2 \mathrm{DEG}$ states. Integration in real space gives

$$
\left|M_{i \boldsymbol{k}_{\|} \rightarrow j \boldsymbol{k}_{\|}^{\prime}}^{2 \mathrm{DEG}}\right|^{2}=\frac{e^{4} e^{-2 q d}\left[\cosh \left(q a_{z}\right)-\cos \left(\boldsymbol{q} \boldsymbol{a}_{\|}\right)\right]}{2 A^{2}\left(\epsilon_{0} \epsilon_{r}\right)^{2}\left(q+\lambda_{2 \mathrm{D}}\right)^{2}},
$$

with the momentum transfer $q=|\boldsymbol{q}|=\left|\boldsymbol{k}-\boldsymbol{k}^{\prime}\right|$.

\section{Three-dimensional electron gas}

Coulomb scattering with semi-confined bulk electrons located at $z>d$ is considered next. The Fourier components of the three-dimensional Poisson equation for this 
problem are

$$
-q^{2} U_{\text {ind }}(q, z)+\partial_{z}^{2} U_{\text {ind }}(q, z)=-\frac{e^{2} \delta n_{3 \mathrm{D}}(q, z)}{\epsilon_{0} \epsilon_{r}} \Theta(z-d),
$$

where the induced bulk electron density is given by

$$
\delta n_{3 \mathrm{D}}(q, z)=-\frac{\partial n_{3 \mathrm{D}}}{\partial E_{F}}\left[U_{\text {ind }}(q, z)+U_{\text {ext }}(q, z)\right] .
$$

With the definition of the Debye screening wave vector

$$
\lambda_{3 \mathrm{D}}=\sqrt{\frac{e^{2}}{\epsilon_{0} \epsilon_{r}} \frac{\partial n_{3 \mathrm{D}}}{\partial E_{F}}}
$$

and the expression for the bulk electron density

$$
n_{3 \mathrm{D}}=2\left(\frac{m^{*} k_{B} T}{2 \pi \hbar^{2}}\right)^{3 / 2} F_{1 / 2}\left(\frac{E_{F}-E_{C}}{k_{B} T}\right)
$$

the Debye screening wave vector is given by

$$
\lambda_{3 \mathrm{D}}=\sqrt{\frac{e^{2}}{\epsilon_{0} \epsilon_{r}} n_{3 \mathrm{D}} \frac{F_{-1 / 2}\left(\frac{E_{F}-E_{C}}{k_{B} T}\right)}{F_{1 / 2}\left(\frac{E_{F}-E_{C}}{k_{B} T}\right)} .}
$$

Here, $F_{s}$ denotes the Fermi integral of order $s$. Solving Eqn. (16) leads to

$$
U_{\text {ind }}(q, z>d)=\frac{-e^{2} e^{-q z}}{2 q \epsilon_{0} \epsilon_{r}}+\frac{e^{2} e^{-q d+\sqrt{\lambda_{3 \mathrm{D}}^{2}+q^{2}}(d-z)}}{\epsilon_{0} \epsilon_{r}\left[q+\sqrt{\lambda_{3 \mathrm{D}}^{2}+q^{2}}\right]} \text {. }
$$

Here, we have used that $U_{\text {ind }}$ is continuously differentiable at $z=d$. Analogously to Eqn. (12) we find the dielectric function for the inhomogeneous bulk electron distribution for $z>d$ as

$$
\epsilon_{e}^{-1}(q, z>d)=\frac{2 q e^{-(z-d)\left(\sqrt{\lambda_{3 \mathrm{D}}^{2}+q^{2}}-q\right)}}{q+\sqrt{\lambda_{3 \mathrm{D}}^{2}+q^{2}}} .
$$

Using continuum wave functions, which are semi-confined in the $z$-direction and plane waves in the radial directions then leads to

$$
\begin{gathered}
\left|M_{i \boldsymbol{k} \rightarrow j \boldsymbol{k}^{\prime}}^{3 \mathrm{DEG}}\right|^{2}=\frac{1}{A^{2}} \mid \int_{d}^{\infty} \mathrm{d} z \sqrt{\frac{2}{L}} \sin \left(k_{z}^{\prime}(z-d)\right) \\
\times \frac{e^{2}}{\epsilon_{0} \epsilon_{r}} \frac{e^{-q d+\sqrt{\lambda_{3 \mathrm{D}}^{2}+q^{2}}(d-z)}}{q+\sqrt{\lambda_{3 \mathrm{D}}^{2}+q^{2}}}\left[e^{q \frac{a_{z}}{2}+i \boldsymbol{q} \frac{\boldsymbol{a}_{\mathrm{u}}}{2}}-e^{-q \frac{a_{z}}{2}-i \boldsymbol{q} \frac{\boldsymbol{a}_{\mathrm{u}}}{2}}\right] \\
\times\left.\sqrt{\frac{2}{L}} \sin \left(k_{z}(z-d)\right)\right|^{2},
\end{gathered}
$$

where $k_{z}$ and $k_{z}^{\prime}$ denote the $z$-components of the wave vectors. Integration over real space results in

$$
\begin{aligned}
& \left|M_{i \boldsymbol{k} \rightarrow j \boldsymbol{k}^{\prime}}^{3 \mathrm{DEG}}\right|^{2}=\frac{2 e^{4} e^{-2 q d}\left[\cosh \left(q a_{z}\right)-\cos \left(\boldsymbol{q} \boldsymbol{a}_{\|}\right)\right]}{(A L)^{2}\left(\epsilon_{0} \epsilon_{r}\right)^{2}} \\
& \frac{\lambda_{3 \mathrm{D}}^{2}+q^{2}}{\left(\sqrt{\lambda_{3 \mathrm{D}}^{2}+q^{2}}+q\right)^{2}}\left[\frac{1}{p_{z}^{2}+\lambda_{3 \mathrm{D}}^{2}+q^{2}}-\frac{1}{q_{z}^{2}+\lambda_{3 \mathrm{D}}^{2}+q^{2}}\right]^{2}
\end{aligned}
$$

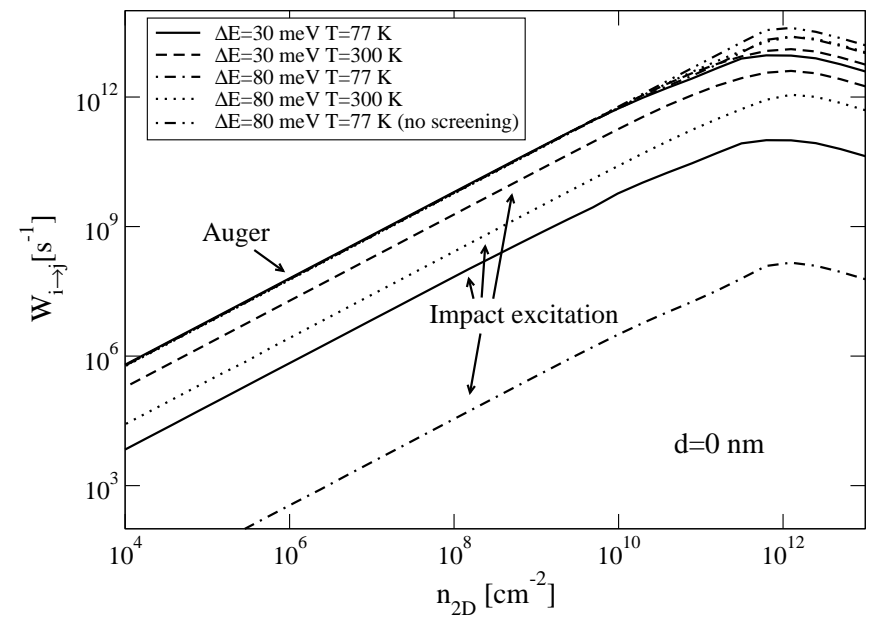

FIG. 2: Auger and impact excitation probabilities as a function of the 2DEG electron density at $d=0 \mathrm{~nm}$ (e.g., for a wetting layer surrounding the QDs) for different energy differences $\Delta E$ and temperatures $T$. In addition the corresponding result without screening is shown for the Auger rate at $\Delta E=80 \mathrm{meV}$ and $77 \mathrm{~K}$ (dash-double-dotted line).

with $q_{z}=k_{z}^{\prime}-k_{z}$ and $p_{z}=k_{z}^{\prime}+k_{z}$. Analogously to the 2DEG, the transition probability is obtained by integration over occupied continuum states, which is performed numerically.

\section{RESULTS}

\section{A. Remote two-dimensional electron gas}

According to the geometry and size of InAs QDs which have a typical base length of about $10 \mathrm{~nm}$ and a strong quantization in z-direction, we use $\left|\boldsymbol{a}_{\|}\right|=5 \mathrm{~nm}$ and $a_{z}=0$. For small dipole moments a Taylor expansion in Eqn. (15) and (24) leads to a quadratic dependence on the transition dipole moments, and the transition probabilities presented here can be rescaled for specifically shaped QDs.

First we performed calculations of the Auger and impact excitation probabilities per unit time as a function of the electron density per unit area in the wetting layer, using $d=0 \mathrm{~nm}$ and $m_{\mathrm{InAs}}^{*}=0.023 m_{0}$ for different QD level spacings $\Delta E=\left|E_{j}-E_{i}\right|$. Fig. [2] shows the resulting probabilities for $\Delta E=30 \mathrm{meV}$ and $80 \mathrm{meV}$ for $\mathrm{T}=77$ $\mathrm{K}$ and $300 \mathrm{~K}$. The Auger probabilities hardly depend on $\Delta E$ and the temperature. For densities less than $10^{10}$ $\mathrm{cm}^{-2}$ the Auger probability is a linear function of the density. From a fit $W_{i \rightarrow j}=T_{\text {Aug }}^{2 \mathrm{D}} n_{2 \mathrm{D}}$ for low densities we obtain an Auger relaxation coefficient of $T_{\text {Aug }}^{2 \mathrm{D}}=60$ $\mathrm{cm}^{2} \mathrm{~s}^{-1}$. This value is about one order of magnitude larger than the corresponding value calculated in Ref. [9] $\left(1 \mathrm{~cm}^{2} \mathrm{~s}^{-1}\right)$. We explain this deviation by the different values for the transition dipole moment and the effective mass used in this work. Note that the Auger capture co- 


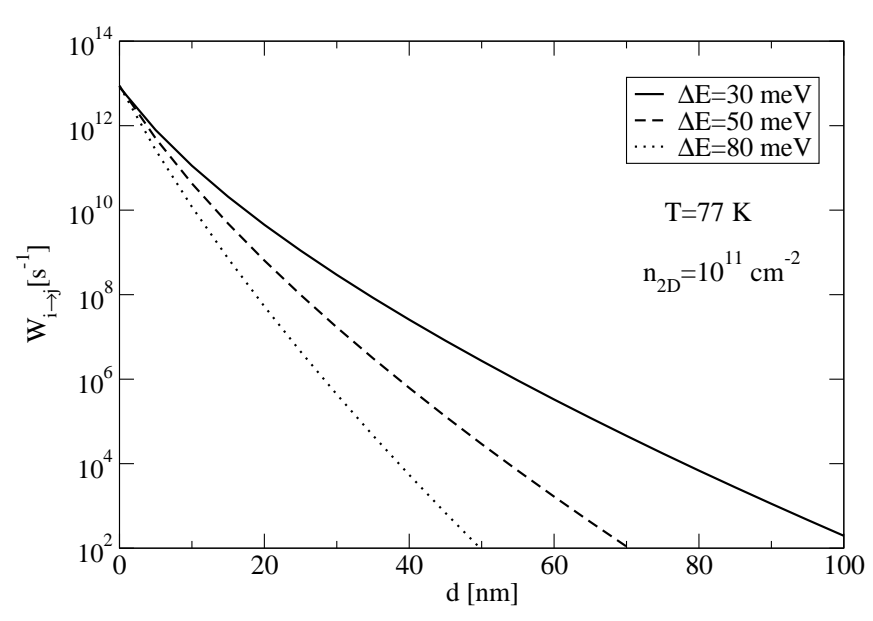

FIG. 3: Remote Auger probabilities as a function of the distance of the 2DEG from the QDs for $n_{2 \mathrm{D}}=10^{11} \mathrm{~cm}^{-2}$, $\Delta E=30 \mathrm{meV}, \Delta E=50 \mathrm{meV}$ and $\Delta E=80 \mathrm{meV}$ at $77 \mathrm{~K}$

efficients evaluated in Ref. [9] $\left(2 \times 10^{-12} \mathrm{~cm}^{4} \mathrm{~s}^{-1}\right)$ are also much smaller than those observed experimentally in Ref. 11] $\left(10^{-8} \mathrm{~cm}^{4} \mathrm{~s}^{-1}\right)$. For large densities, i.e., when the 2DEG becomes degenerate, the density dependence becomes nonlinear, and the probabilities are even reduced.

Fig. 2 also shows that the impact excitation probabilities strongly depend on $\Delta E$ and $T$, and the ratio between the impact excitation and Auger probabilities is given by the Boltzmann factor $\exp \left(-\Delta E /\left(k_{B} T\right)\right)$. The Auger probability for $\Delta E=80 \mathrm{meV}$ and $\mathrm{T}=77 \mathrm{~K}$ but neglecting screening effects is also plotted in Fig. 2 demonstrating that screening effects play only a role for densities typically larger than $10^{10} \mathrm{~cm}^{-2}$.

Fig. 3] shows the remote Auger probabilities as a function of the distance $d$ between the QDs and the 2DEG, for $n_{2 \mathrm{D}}=10^{11} \mathrm{~cm}^{-2}, m_{\mathrm{GaAs}}^{*}=0.06 m_{0}, T=77 \mathrm{~K}$ and $\Delta E=30 \mathrm{meV}, \Delta E=50 \mathrm{meV}$ and $\Delta E=80 \mathrm{meV}$. The Auger probabilities decrease exponentially with $d$, depending on the energy transfer. Therefore the Coulomb scattering of this type has a local character. As a consequence, a $2 \mathrm{DEG}$ at $d=200 \mathrm{~nm}$ as in the memory structure of Ref. [18] does not contribute to the lifetime limitation of charged QDs.

\section{B. Remote three-dimensional electron gas}

Next, we calculated the Coulomb scattering probabilities as a function of the bulk density in the vicinity of the QDs. The result is plotted for $\Delta E=30 \mathrm{meV}$ (solid line), $\Delta E=50 \mathrm{meV}$ (dashed line) and $\Delta E=80 \mathrm{meV}$ (dotted line) in Fig. 4 for $\mathrm{T}=77 \mathrm{~K}, d=0 \mathrm{~nm}$ and $m_{\mathrm{GaAs}}^{*}=0.06$ $m_{0}$. Again, for low densities the probabilities are linear in the density. But in comparison to the 2DEG, the fitted Auger coefficients $T_{\text {Aug }}^{3 \mathrm{D}}=W_{i \rightarrow j} / n_{3 \mathrm{D}}$ depend on the QD level spacings. Here we obtain $T_{\text {Aug }}^{3 \mathrm{D}}=4.7 \times 10^{-6} \mathrm{~cm}^{3} \mathrm{~s}^{-1}$, $T_{\text {Aug }}^{3 \mathrm{D}}=1.9 \times 10^{-6} \mathrm{~cm}^{3} \mathrm{~s}^{-1}$ and $T_{\text {Aug }}^{3 \mathrm{D}}=1.0 \times 10^{-6} \mathrm{~cm}^{3} \mathrm{~s}^{-1}$ for

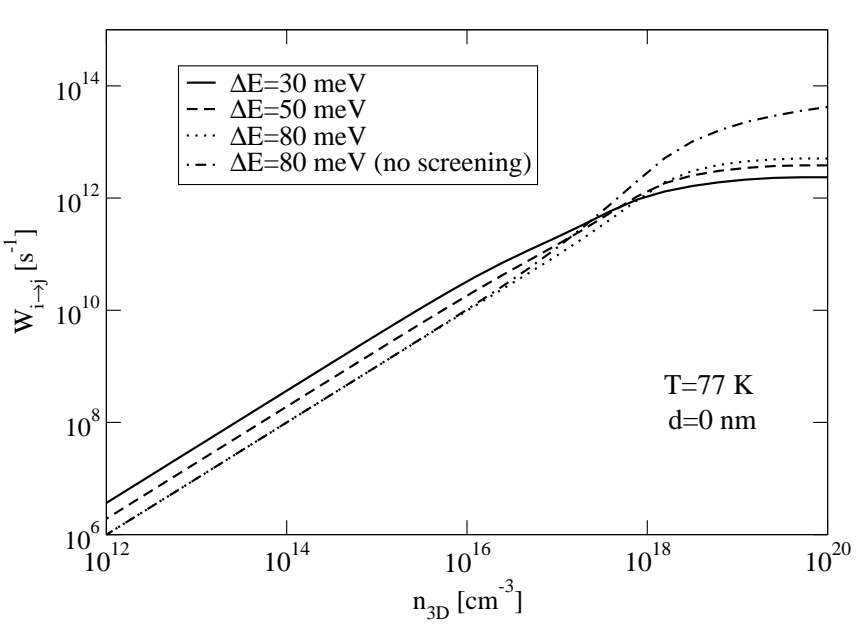

FIG. 4: Auger probabilities as a function of the threedimensional electron density for different energy level separations $\Delta E$ at $77 \mathrm{~K}$ and with $d=0 \mathrm{~nm}$. In addition the corresponding result without screening is shown for the Auger rate at $\Delta E=80 \mathrm{meV}$ (dash-dotted line).

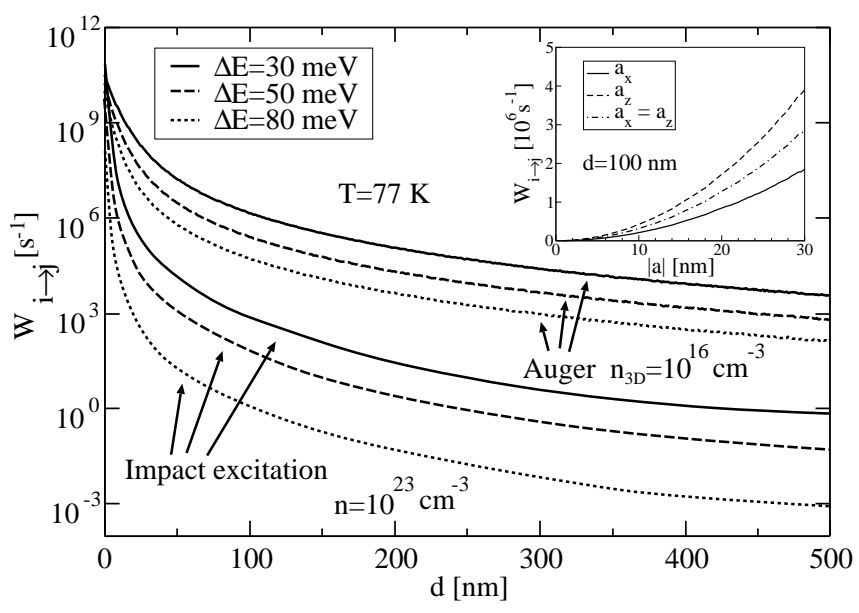

FIG. 5: The Auger probabilities for a three-dimensional electron gas with $n_{3 \mathrm{D}}=10^{16} \mathrm{~cm}^{-3}, \mathrm{~m}^{*}=0.06 \mathrm{~m}_{0}$ and the impact excitation probabilities for $n_{3 \mathrm{D}}=10^{23} \mathrm{~cm}^{-3}, \mathrm{~m}^{*}=m_{0}$ as a function of $d$ for different level spacings $\Delta E$ at $77 \mathrm{~K}$. Inset: dependence of the Auger probability for $n_{3 \mathrm{D}}=10^{16} \mathrm{~cm}^{-3}$, $m^{*}=0.06 m_{0}$ and $\Delta E=80 \mathrm{meV}$ on the transition dipole moment for $d=100 \mathrm{~nm}: a_{x} \neq 0, a_{z}=0$ (solid line); $a_{x}=0$, $a_{z} \neq 0$ (dashed line) and $a_{x}=a_{z} \neq 0$ (dash-dotted line)

$\Delta E=30 \mathrm{meV}, \Delta E=50 \mathrm{meV}$ and $\Delta E=80 \mathrm{meV}$, respectively. For high electron densities we observe a deviation from this linear behavior, since pairs of occupied and empty continuum states can only be found near the Fermi level when the electron gas becomes highly degenerate. The resulting Auger probability neglecting screening effects for $\Delta E=80 \mathrm{meV}$ is plotted as a dash-dotted line in Fig. 4 demonstrating that screening is only important for densities above $10^{17} \mathrm{~cm}^{-3}$.

The Auger probability as a function of the distance from the QDs is investigated next. The resulting curves 


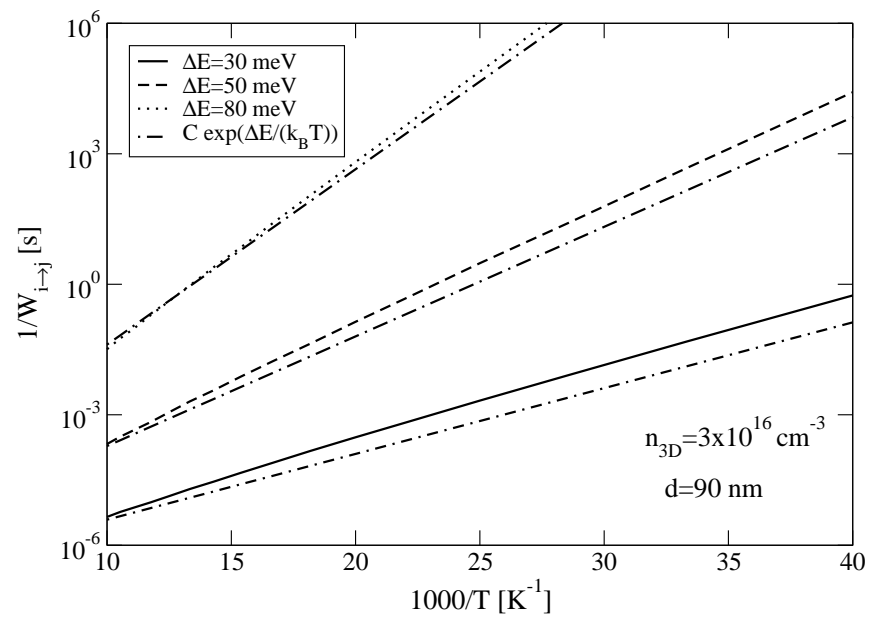

FIG. 6: Arrhenius plot of the inverse impact excitation probability with $d=90 \mathrm{~nm}, n_{3 \mathrm{D}}=3 \times 10^{16} \mathrm{~cm}^{-3}$ for $\Delta E=30 \mathrm{meV}$ (solid line), $\Delta E=50 \mathrm{meV}$ dashed line, $\Delta E=80 \mathrm{meV}$ (dotted line); dash-dotted lines: functions proportional to the Boltzmann factor $C \exp \left(\Delta E /\left(k_{B} T\right)\right)$ where $C$ is chosen such that lines intersect at $1000 / T=10 \mathrm{~K}^{-1}$

for $n_{3 \mathrm{D}}=10^{16} \mathrm{~cm}^{-3}, m_{\mathrm{GaAs}}^{*}=0.06 m_{0}, \Delta E=30 \mathrm{meV}$ (solid line), $\Delta E=50 \mathrm{meV}$ dashed line, $\Delta E=80 \mathrm{meV}$ (dotted line) at $77 \mathrm{~K}$ are shown in Fig. 5. Compared to the remote 2DEG scattering, the dependence on $d$ is weaker, since momentum in the 3DEG can additionally be changed in the third dimension. An interesting point is how a metal contact with its high electron density limits the lifetime of charged QDs. Therefore, the impact excitation probabilities are also displayed in Fig. 5 for $n_{3 \mathrm{D}}=10^{23} \mathrm{~cm}^{-3}$, which is a typical electron density in metal contacts, and $m^{*}=m_{0}$. Depending on the energy spacing $\Delta E$, we obtain transition times of the order of an hour for $\Delta E=80 \mathrm{meV}$ and $d=400 \mathrm{~nm}$. This goes well with the observation of bistable behavior in QD structures on this time scale [18, 19]. The inset of Fig. [5 shows the dependence of the Auger probability on the transition dipole moment $-e \boldsymbol{a}$, which can be well approximated by a quadratic law resulting from the Taylor expansion of Eq. (23). The Taylor expansion also explains that the transition probabilities are enlarged by a factor of 2 for vertical dipole moments (in growth direction) compared to the lateral dipole moments studied throughout this work. We conclude that the direction of the dipole moment does not affect the order of magnitude of the transition rates.

Finally we consider QDs embedded in a pn diode, where the QDs are positioned within $3 \times 10^{16}$ n-doped
GaAs layers. Such a device was investigated in DLTS experiments, and a level spacing of $\Delta E=82 \mathrm{meV}$ with an emission time of $62 \mathrm{~ms}$ at $\mathrm{T}=40 \mathrm{~K}$ has been observed [14]. The emission from the QD ground state level is dominated by an excitation into the excited state, from which the electron escapes into the continuum by a fast tunneling process. Taking into account the Boltzmann factor between excitation and relaxation processes we estimate a timescale for the relaxation process from the excited state into the ground state of about 3 ps. From Fig. 2 we conclude that for an Auger process on that fast time scale a wetting layer density of $10^{10} \mathrm{~cm}^{-2}$ would be necessary. However, the wetting layer is not occupied due to the electric field in the depletion layer. From the parabolic band bending and a QD ground state energy of $200 \mathrm{meV}$ below the conduction band edge we estimate that the depletion layer extends to $d=90 \mathrm{~nm}$ from the QDs. For these parameters, we display the temperature dependence as an Arrhenius plot as shown in Fig. 6 for $\Delta E=30 \mathrm{meV}$ (solid line), $\Delta E=50 \mathrm{meV}$ dashed line, $\Delta E=80 \mathrm{meV}$ (dotted line). The Botzmann factor (functions proportional to $\exp \left(\Delta E /\left(k_{B} T\right)\right)$ chosen such that the lines intersect at $\left.1000 / T=10 \mathrm{~K}^{-1}\right)$ is also displayed as dash-dotted line for each energy spacing, showing that the slope of the Arrhenius plot of the temperature dependence is mainly given by the energy spacing.

Nevertheless, we obtain an impact excitation time of about $10^{4} \mathrm{~s}$ for $T=40 \mathrm{~K}$ and $\Delta E=80 \mathrm{meV}$, which is orders of magnitudes slower than observed in the DLTS experiment. Only by reducing the level spacing below $\Delta E=50 \mathrm{meV}$ impact excitation times in the range of tens of ms can be obtained at this temperature. This indicates that in addition phonon mechanisms have to be taken into account.

\section{CONCLUSION}

In conclusion, we have evaluated the Auger effect and impact excitation (Coulomb scattering) in QD devices with remote continuum states. Three-dimensional Debye screening has been included, which is important for high electron densities. Our results show that, depending on the detailed device and QD geometries, scattering with remote continuum carriers can be of importance for the electron kinetics if the local wetting layer density is reduced.

This work was supported by DFG in the framework of Sfb 296. Helpful discussions with M. Geller and R. Heitz are acknowledged.
[1] D. Bimberg, M. Grundmann, and N. Ledentsov, Quantum Dot Heterostructures (John Wiley \& Sons Ltd., New York, 1999).

[2] R. Heitz, T. R. Ramachandran, A. Kalburge, Q. Xie,
I. Mukhametzhanov, P. Chen, and A. Madhukar, Phys. Rev. Lett. 78, 4071 (1997).

[3] I. Magnusdottir, A. V. Uskov, S. Bischoff, B. Tromborg, and J. Mørk, J. Appl. Phys. 92, 5982 (2002). 
[4] J. Feldmann, S. T. Cundiff, M. Arzberger, G. Böhm, and G. Abstreiter, J. Appl. Phys. 89, 1180 (2001).

[5] I. Magnusdottir, A. V. Uskov, R. Ferreira, G. Bastard, J. Mørk, and B. Tromborg, Appl. Phys. Lett. 81, 4318 (2002).

[6] O. Verzelen, R. Ferreira, and G. Bastard, Phys. Rev. B 62, 4809 (2000).

[7] U. Bockelmann and G. Bastard, Phys. Rev. B 42, 8947 (1990).

[8] U. Bockelmann and T. Egeler, Phys. Rev. B 46, 15574 (1992).

[9] A. V. Uskov, J. McInerney, F. Adler, H. Schweizer, and M. H. Pilkuhn, Appl. Phys. Lett. 72, 58 (1998).

[10] A. V. Uskov, F. Adler, H. Schweizer, and P. H. Kuhn, J. Appl. Phys. 81, 7895 (1997).

[11] S. Raymond, K. Hinzer, S. Fafard, and J. L. Merz, Phys. Rev. B 61, 16331 (2000).

[12] D. Morris, N. Perret, and S. Fafard, Appl. Phys. Lett.
75, 3593 (1999).

[13] P. W. Fry, J. J. Finley, L. R. Wilson, A. Lemaitre, D. J. Mowbray, M. S. Skolnick, M. Hopkinson, G. Hill, and J. C. Clark, Appl. Phys. Lett. 77, 4344 (2000).

[14] C. M. A. Kapteyn, M. Lion, R. Heitz, D. Bimberg, P. Brunkov, B. Volovik, S. Konnikov, A. Kovsh, and V. Ustinov, Appl. Phys. Lett. 76, 1573 (2000).

[15] R. Wetzler, C. M. A. Kapteyn, R. Heitz, A. Wacker, E. Schöll, and D. Bimberg, Appl. Phys. Lett. 77, 1671 (2000).

[16] R. Wetzler, A. Wacker, and E. Schöll, Phys. Rev. B (2003), in print.

[17] P. T. Landsberg, Recombination in Semiconductors (Cambridge University Press, Cambridge, 1991).

[18] G. Yusa and H. Sakaki, Appl. Phys. Lett. 70, 345 (1997).

[19] A. Rack, R. Wetzler, A. Wacker, and E. Schöll, Phys. Rev. B 66, 165429 (2002). 